Open Access

\title{
A study of the influential factors for health inequality
}

Kaishan Jiao

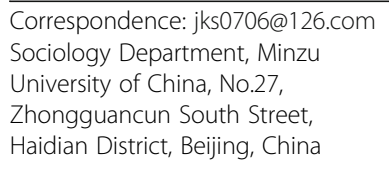

Correspondence: jks0706@126.com Sociology Department, Minzu University of China, No.27, Zhongguancun South Street, Haidian District, Beijing, China

\begin{abstract}
Using a national dataset, this study investigates the inequalities in physical functional status, depressive symptoms, and self-rated health among groups of people with different socioeconomic status (SES) through the use of multilevel regression models. The results reveal that as age increases, differences in physical functional status among people with different SES expand and no significant differences in depressive symptoms are found. However, general conclusions on the trend of differences in self-rated health cannot be made. In wealthy regions, the differences in the three health indicators among people with different SES shrink, but these differences expand in poor regions. Finally, this study advocates that the emphasis of the structural reform of medical treatment and public health should be elderly with low SES who resides in poor regions.

Keywords: Socioeconomic status (SES), Health inequality, Expand, Shrink
\end{abstract}

\section{Introduction}

Due to its profound intrinsic value, health is usually regarded as an important criterion to evaluate the development of a society. However, health differences among different groups of people are an objective reality. Health inequality arises when there are avoidable differences in health or differences in major social determinants for health among groups of people with different social advantages (wealth, power, or social status). Health inequality is an important part of social inequality and presents a serious challenge (Guo and Xie 2009) in both wealthy and impoverished countries, thus becoming a major research topic in the social sciences over the past 20 or 30 years.

Although the majority of previous research indicates significant associations between socioeconomic status (SES) and health (e.g., Feinstein 1993; Mackenbach et al. 1997; Wang 2011), the conclusions were based primarily on adult samples, and no consensus for the elderly group has been reached. Some research suggests that the relationship between SES and health would be strengthened among the elderly, but others point out that the relationship could be weakened. In addition, most previous studies were based on people from developed countries, and their conclusions may not be applicable to people in developing countries since there are significant differences in the level of economic development, social systems, and population structure between developing and developed countries. For example, the SES-health relationship may present different forms between different countries or areas. Given the above considerations, the

(c) Jiao. 2016 Open Access This article is distributed under the terms of the Creative Commons Attribution 4.0 International License (http://creativecommons.org/licenses/by/4.0/), which permits unrestricted use, distribution, and reproduction in any medium, provided you give appropriate credit to the original author(s) and the source, provide a link to the Creative Commons license, and indicate if changes were made. 
current study focuses on the middle-aged and elderly groups in China, investigating the potential differences in the SES-health relationship between the two groups. Does the difference in health among groups of people with different SES expand or shrink during the elderly stage? Is there a significant difference in the SES-health relationship among different regions, that is, does the health difference among groups of people with different SES get larger or smaller as the area becomes wealthier?

\section{Literature review and research question}

Ever since the release of the "Black Report" (Black 1981), health inequality has received increasing attention in academic research. The research indicates that social stratification by health exists in almost all societies, i.e., the average health of people with high SES is better than that of people with low SES (Feinstein 1993; Mackenbach et al. 1997). The following factors constitute health advantages for high-SES groups: low risk of health impairment due to superior working and living conditions (Evans and Kantrowitz 2002; Liu and Tang 2004), advantages in acquiring health care knowledge and in making use of medical information and technology as a result of superior educational background (Glied and Lleras-Muney 2008), advantages in accessing and utilizing medical treatment and public health resources and services (Victora et al. 2000; Xie 2009; Meng 2007), and healthier lifestyles (Wang 2012). Nevertheless, health differences among different SES groups may be the consequence of "selection," i.e., healthier people tend to attain high SES while less healthy people tend to move to the lower social classes (Dahl 1996; West 1991; Wang 2011), hence leading to greater differences in health among different SES groups.

Although the positive correlation between SES and health has already been well established, no consensus on how this correlation may change with age has been ascertained. Previous studies found that the health differences among different SES groups grew larger prior to the initial period of the middle-aged and elderly stage, but the differences decreased during the elderly period (Beckett 2000; House et al. 1990, 1994). Some researchers define this argument as the "Convergence Hypothesis" (Lowry and Xie 2009). The reason that health differences gradually become smaller during the elderly stage may be that in the elderly stage, the differences in psychological and social risk factors (such as a lack of social relations and social support or the deprivation of the feeling of being in control) faced by people with different SES gradually reduced or even disappeared (House et al. 1994; Lantz et al. 1998). However, the explanation may be the gradual enhancement of the determining effects of biological factors on health; these determining effects may even dominate the effects of socioeconomic factors (Mirowsky and Ross 2008). However, a large number of research works indicate that the effect of SES on health accumulates through the entire human life process; instead of shrinking, health differences among groups of people with different SES expand as age increases, and health inequality during the elderly stage is more severe than during the middle-aged stage (Dupre 2008; Lowry and Xie 2009; Lynch 2003; Mirowsky and Ross 2005; Ross and Wu 1996). Some researchers label this argument as the "Cumulative Advantage Hypothesis" (Lowry and Xie 2009). To summarize, no consensus has been reached on the relationship between SES and health during the elderly stage, and the most current conclusions are 
based on the empirical evidence from developed countries, which demonstrates the need for ongoing research on this problem in a different socioeconomic setting such as China.

Previous research has pointed out that the SES-health relationship is affected by a country's social, political, and economic conditions (Lowry and Xie 2009), that is, regional socioeconomic conditions can change the mechanism by which individual SES relates to health. At the country level, some research indicates that the effects of individual SES on health are stronger in developed countries because socioeconomic factors have become the major determinant for health in these countries (Wilkinson 1997). Other research indicates that although the rate of mortality and morbidity among people with low SES was higher in all Western European countries, the inequalities were greater in Sweden and Norway, whereas the differences in the mortality rate among people with different SES in France were the highest among all the Western European countries (Mackenbach et al. 1997). No significant differences in the level of health inequalities were detected among people of different social classes, while differences in the level of inequalities by gender prevailed in Latin America and Caribbean countries (Dachs et al. 2002). In Asia, differences in the effects of SES on health were found within Thailand and Philippines (Zimmer et al. 2004). Differences in the effects of SES on health can occur in different regions of the same country. Since the socioeconomic background of a region has a profound impact on individual health (Pickett and Pearl 2001; Robert 1998; Yen and Syme 1999), the degree to which individual SES and health are associated can be adjusted (Bassuk et al. 2002), thereby making a difference in the impact on groups of people with different SES (Robert 1998). Therefore, if an individual with low SES lives in an area with poor socioeconomic conditions, the health risk factors could double: heath differences among people with different SES could increase in a region with poor socioeconomic conditions; in contrast, if an individual with low SES lives in a region with improved socioeconomic conditions, the resulting feelings of deprivation may have a negative impact on health (Ellaway et al. 2012), therefore widening the gap between the health of different SES groups.

In addition, previous research indicates that the SES-health relationship may differ in different health indicators (Huurre et al. 2005), resulting from the differences in the connotations, properties of the measure of each health indicator, and effects of the external social factors. In social science research, the measuring indicators of health conditions include self-rated health, physical functional status, incidence of disease, and depressive symptoms, among others. Physical functional status and the incidence of disease are relative objective indicators, which are more sensitive in response to socioeconomic factors (Sun et al. 2003). For instance, the research finds that health disparities caused by the socioeconomic status is the largest for some of the most preventable diseases (Phelan et al. 2004). Self-rated health is among the most commonly used and most popular health indicators and is a more tolerant, accurate measure of health conditions and risk factors (Idler and Benyamini 1997). Unlike most health indicators, however, self-rated health relies on the process of subjective cognition. Self-rated health is not only affected by the objective health conditions of an individual but also influenced by individual feelings, cognitive framework, and socioeconomic background. It is an interactive and constructive process involving the objective health condition and the 
subjective cognition (Jylhä 2009). Differences in self-rated health may not be in a one-toone relationship with the "objective" difference in health among different groups of people (Dowd and Zajacova 2010). For example, individuals with low literacy or from areas with poor medical treatment and public health conditions are likely to report better health conditions than they actually had because of their lack of knowledge about their risks (Dowd and Zajacova 2010). Most elderly people have a positive attitude toward their health condition; even those who live in nursing homes have positive ratings for their health conditions (Hooyman and Kiyak 2011). As a result, compared to the differences in some objective health indicators, the differences in self-rated health among people with different SES are relatively smaller, especially among the elderly group.

From the above literature review, we find that previous studies on health inequalities mostly targeted developed countries; only a few studies were based on health inequalities in China and they appeared quite recently. The question of whether the consensus obtained from empirical evidence in Western developed countries can be applied to China merits further investigation. In the meantime, we address whether health differences among people with different SES expand or shrink during the elderly stages. Ongoing research on this problem in the context of China's socioeconomic background could provide new empirical evidence to resolve this question. Additionally, although previous studies took into account the effects of socioeconomic factors on individual health conditions, little has been put forward on whether significant health differences exist in different areas among people with different SES. Finally, the majority of the previous research uses a single health measurement indicator, which overlooks the multidimensionality and totality of health.

Based on the above considerations, this study explores the relationship between SES and different health measurement indicators. Through the use of multilevel data, it examines whether significant differences in the relationship exist among people of different age groups or from different regions. Specifically, this study focuses on the following problems:

1. Although the socioeconomic background in China is quite different from that in developed countries, several factors, including the unbalanced development of urban and rural areas, wider income gaps between people, an incomplete medical insurance system, and the gradual social structure differentiation since the implementation of the reform and open policy, aggravate health inequalities in China. The first research question is thus to further ascertain whether significant differences exist among Chinese people aged over 45 in physical functional status, levels of depression, and self-rated health across different groups of people with different SES.

2. This study investigates whether significant differences occur due to age groups among the health differences of people with different SES. Specifically, will the gap between the health of different SES groups expand or shrink in the elderly stage? Does the age pattern of the SES-health relationship differ with different choices of health indicators?

3. In view of the fact that the areal social and economic conditions are not only important influential factors for the individual's health but also related to the individual's SES, this study further examines the areal pattern of the SES-health 
relationship. Across areas with different levels of wealth, will the pattern of SEShealth relationship be different? With the improvement in an area's wealth, will the gap between the health of different SES groups expand or shrink?

\section{Data and statistical methods for analysis}

(i) Data

The data was obtained from the national baseline survey in the "China Health and Retirement Longitudinal Study (CHARLS)” during 2011. ${ }^{1}$ A random sample was taken among individuals in the family aged over 45 . The CHARLS baseline survey covers 450 villages and neighborhoods in 150 counties or districts across the country. In addition to the data from individuals and their family members, the study collected information on the communities. After deleting the cases not eligible to the study purpose, the sample size of the study was 12,246 , consisting of individuals from 149 county-level units.

(ii) Variables and method of measurement

Three dependent variables were collected in the study: physical functional status, depressive symptoms, and self-rated health. Physical functional status includes the activities of daily living (ADL) and instrumental activities of daily living (IADL), encompassing seventeen activities of daily living in total. ${ }^{2}$ Each of the activities had four possible responses: (1) with no difficulty, (2) with difficulty but can still be completed, (3) with difficulty and need help, and (4) cannot be completed. The four choices were assigned the values of one through four; the total score of physical functional status for each person was obtained by summing component scores across the 17 activities. This study treated the total score as a continuous variable ranging from 17 to 68 ; higher scores indicate worse physical activity status.

In CHARLS, the depressive symptoms of respondents are measured based on ten items, nine of which are included in the current study. ${ }^{3}$ Each item consists of four choices: (1) rarely or none, (2) not too much, (3) sometimes or half of the time, and (4) most of the time. We assigned each choice a value ranging from one to four and obtained the total score of depressive symptoms for each individual by summing the component scores over nine activities. The study regards the total score of depressive symptoms as a continuous variable ranging from nine to 36 , with higher scores indicating worse depressive symptoms.

Self-rated health measures the respondents' answers to the question, "How do you consider your current health condition?" We grouped the answers "very good," "good," and "ordinary" as one category ${ }^{4}$, namely self-rated "good" (coded as zero), and the answers "not good" and "very bad" as another category, self-rated "not good" (coded as one).

The explanatory variable in this study is SES. Education, occupation, and income are commonly used measures of SES, but some studies indicate such measurements are primarily applicable to developed countries; whether they can be applied to developing countries merits further study (Zhu and Xie 2007). Given the influence of the specific household registration system in China, we also regard the household register as an indicator of SES. Although treating the household register as an indicator for SES is controversial, we agree with studies indicating 
that household register forms the most important determinant for the distribution of social resources and power (Wu and Treiman 2004), justifying its use as a measurement of SES (Zhu and Xie 2007). In the present study, household register is divided into two categories: (1) rural household register and (2) urban household register. Educational attainment is classified into four categories: (1) illiteracy, (2) elementary school level ${ }^{5}$, (3) junior high school level, and (4) high school level or above. Since the occupations among the middle-aged and elderly, Chinese are not dispersed, a fair number of zero counts would be expected if we divided the occupation into more categories, which in turn affects the model estimates. Therefore, we combined some occupation categories and obtained two major categories ${ }^{6}$ : (1) physical workers, including farmers and workers, and (2) nonphysical workers, including management, professional and technical personnel, officers, and business services personnel. The main occupation before retirement was recorded if the respondent had retired. In the current study, income was classified into four categories $^{7}$ : annual household income per capita falling (1) below 2300 yuan, (2) between 2300 and 5000 yuan, (3) between 5000 and 10,000 yuan, and (4) above 10,000 yuan. Given the high correlations among these SES variables, we obtained a general evaluation of SES using the latent class analysis (LCA) method. By comparing goodness-of-fit statistics for different models, we identified the optimal model with three latent classes. In other words, given the chosen education, income, occupation, and household variables, respondents could be classified into three groups: high, medium, and low socioeconomic status.

In addition to individual SES, area income level was investigated as an important socioeconomic factor. We used the median household income per capita to measure the area income level, representing the degree of wealth in an area. We also included sex, age, marital status, health behaviors, and other variables in the present study.

(iii) Analysis methods

For the two dependent variables of physical functional status and depressive symptoms, the study used multilevel linear regression models. For the dependent variable, self-rated health, we used multilevel models for binary responses. The basic strategy was moving from simple models to more complex models and from a few variables to more. We started with a simple model and gradually incorporated relevant independent variables. We first investigated the fixed components of the model and then added random components. We began with the lowest level and moved to higher levels.

Above all, we fit a random intercept model with no explanatory variables to examine whether there were significant cluster effects, i.e., model 1.

$$
\begin{aligned}
& \mathrm{y}_{i j}=\beta_{0}+\mu_{0 j}+e_{i j} \\
& \quad \text { or } \\
& \log \left(\frac{\pi_{i j}}{1-\pi_{i j}}\right)=\beta_{0}+\mu_{0 j}
\end{aligned}
$$

where $i$ denotes a level-one unit, namely the respondent; $j$ denotes a level-two unit, namely a county-level unit; $y_{i j}$ is the score of physical functional status or 
depressive symptoms; $\pi_{i j}$ is the probability of self-rated health as "not-good"; and $\mu_{0 j}$ denotes the residual of the level-two unit, representing the area effect.

Second, we added individual-level explanatory variables to model 1, including sex, age, marital status, and smoking, and obtained model 2. We incorporated individual-level SES variables into model 2, i.e., dummy variables representing medium and highest SES and obtained model 3. Model 4 was obtained by adding interaction terms between SES and age in model 3. Model 5 was obtained from model 4 with additional level-two explanatory variables, namely area income level. On the basis of model 5, model 6 considers the random effects due to SES variables, namely a random coefficient model in which the relationship between SES and dependent variables can vary in different level-two units. Based on model 6, cross-level interaction terms, namely the interaction of SES variables and area income level for investigating whether SES-health relationships are affected by the area income level variable, were added to produce model 7. Model 7 is written as:

$$
\begin{aligned}
y_{i j}=\beta_{0} & +\beta_{1} \operatorname{ses}_{2 i j}+\beta_{2} \operatorname{ses}_{3 i j}+\beta_{3} \operatorname{ses}_{2 i j} \times \operatorname{age}_{i j}+\beta_{4} \operatorname{ses}_{3 i j} \times \operatorname{age}_{i j} \\
& +\beta_{5} \operatorname{ses}_{2 i j} \times \text { countyinc }_{j}+\beta_{6} \operatorname{ses}_{3 i j} \times \text { countyinc }_{j} \\
& +\sum_{k=7}^{p} \beta_{k} x_{k i j}+\left(u_{0 j}+u_{1 j} \operatorname{ses}_{2 i j}+u_{2 j} \operatorname{ses}_{3 i j}+e_{0 i j}\right)
\end{aligned}
$$

or

$$
\begin{aligned}
\log \left(\frac{\pi_{i j}}{1-\pi_{i j}}\right)=\beta_{0} & +\beta_{1} \operatorname{ses}_{2 i j}+\beta_{2} \operatorname{ses}_{3 i j}+\beta_{3} \operatorname{ses}_{2 i j} \times \operatorname{age}_{i j}+\beta_{4} \operatorname{ses}_{3 i j} \times \text { age }_{i j} \\
& +\beta_{5} \operatorname{ses}_{2 i j} \times \operatorname{countyinc}_{j}+\beta_{6} \operatorname{ses}_{3 i j} \times \text { countyinc }_{j} \\
& +\sum_{k=7}^{p} \beta_{k} x_{k i j}+\left(u_{0 j}+u_{1 j} \operatorname{ses}_{2 i j}+u_{2 j} \operatorname{ses}_{3 i j}\right)
\end{aligned}
$$

where $\operatorname{ses}_{2 i j}$ and $\operatorname{ses}_{3 i j}$ variables are two dummy variables, denoting medium and highest SES; countyinc $i$ denotes area income level; $\beta_{3} \operatorname{ses}_{2 i j} \times \operatorname{age}_{i j}$ and $\beta_{4} \operatorname{ses}_{3 i j} \times \operatorname{age}_{i j}$ denote the interaction terms between SES and age; $\operatorname{ses}_{3 i j} \times$ countyinc $_{i}$ denotes the cross-level interaction, i.e., the interaction between SES and area income level; the coefficients of $\operatorname{ses}_{2 i j}$ and $\operatorname{ses}_{3 i j}$ are random at level two; and the intercept is treated as a random variable. The covariance matrix for the intercept and the coefficients, denoted by $\Omega_{2}$, often needs to be computed. The term $\Omega_{1}$ is used to represent the covariance matrix at level one and comes with a single variance term at level one.

$$
\Omega_{2}=\left(\begin{array}{ccc}
\sigma_{u 0} & \sigma_{01} & \sigma_{02} \\
\sigma_{01} & \sigma_{u 1} & \sigma_{12} \\
\sigma_{02} & \sigma_{12} & \sigma_{u 2}
\end{array}\right) \quad \Omega_{1}=\sigma_{e 0}^{2}
$$

In general, the estimation for multilevel linear regression models usually proceeds with the maximum likelihood method. Comparison of nested model proceeds with $-2 \mathrm{LL}$, the deviance statistic for significance testing. We estimated all model parameters using the lme4 packages available in the R statistical software. ${ }^{8}$ 


\section{Results}

(i) Comparison of model goodness-of-fit

Table 1 presents the log likelihood for different models and the results from the likelihood ratio test. The model structures grow more complex from model 0 to model 7; these are all nested models. Model 0 is a simple intercept model without effects from higher-level units (county level in the present study). Models 1 to 7 are two-level regression models, namely models with area effects. Of these, model 1 is the simplest model of all two-level regression models, namely the random intercept model. The comparison of the likelihood ratio of model 1 versus model 0 reveals that there is a significant effect on health conditions due to area characteristics. Therefore, we must consider multilevel models with area effects. Compared to model 1, model 2 has additional control variables, including sex, age, marital status, and smoking. We observe that

Table 1 Comparison of model goodness-of-fit and corresponding likelihood ratio test

\begin{tabular}{|c|c|c|c|c|c|c|}
\hline & $d f$ & Likelihood & Deviance & Chi-square & Chi-square $d f$ & $P$ \\
\hline \multicolumn{7}{|c|}{ Physical functional status } \\
\hline Model 0 & 2 & $-40,079$ & 80,158 & & & \\
\hline Model 1 & 3 & $-39,933$ & 79,867 & 290.900 & 1 & $.000^{* * *}$ \\
\hline Model 2 & 9 & $-39,169$ & 78,338 & 1528.300 & 6 & $.000^{* * *}$ \\
\hline Model 3 & 11 & $-39,123$ & 78,246 & 91.860 & 2 & $.000^{* * *}$ \\
\hline Model 4 & 13 & $-39,109$ & 78,218 & 28.485 & 2 & $.000^{* * *}$ \\
\hline Model 5 & 14 & $-39,105$ & 78,210 & 8.256 & 1 & $.004^{* *}$ \\
\hline Model 6 & 19 & $-39,079$ & 78,157 & 52.573 & 5 & $.000^{* * *}$ \\
\hline Model 7 & 21 & $-39,076$ & 78,153 & 4.222 & 2 & .121 \\
\hline \multicolumn{7}{|c|}{ Depressive symptoms } \\
\hline Model 0 & 2 & $-39,165$ & 78,330 & & & \\
\hline Model 1 & 3 & $-38,652$ & 77,303 & 1027.120 & 1 & $.000^{* * *}$ \\
\hline Model 2 & 9 & $-38,333$ & 76,666 & 636.895 & 6 & $.000^{* * *}$ \\
\hline Model 3 & 11 & $-38,239$ & 76,479 & 187.558 & 2 & $.000^{* * *}$ \\
\hline Model 4 & 13 & $-38,239$ & 76,477 & 1.313 & 2 & .519 \\
\hline Model 5 & 14 & $-38,224$ & 76,448 & 29.112 & 1 & $.000^{* * *}$ \\
\hline Model 6 & 19 & $-38,200$ & 76,400 & 47.904 & 5 & $.000^{* * *}$ \\
\hline Model 7 & 21 & $-38,191$ & 76,382 & 18.701 & 2 & $.000^{* * *}$ \\
\hline \multicolumn{7}{|c|}{ Self-rated health } \\
\hline Model 0 & 1 & -7348 & 14,695 & & & \\
\hline Model 1 & 2 & -7215 & 14,429 & 266.016 & 1 & $.000^{* * *}$ \\
\hline Model 2 & 8 & -7042 & 14,083 & 346.010 & 6 & $.000^{* * *}$ \\
\hline Model 3 & 10 & -6980 & 13,960 & 122.719 & 2 & $.000^{* * *}$ \\
\hline Model 4 & 12 & -6979 & 13,958 & 2.408 & 2 & .300 \\
\hline Model 5 & 13 & -6968 & 13,936 & 21.521 & 1 & $.000^{* * *}$ \\
\hline Model 6 & 18 & -6966 & 13,933 & 3.753 & 5 & .586 \\
\hline Model 7 & 20 & -6961 & 13,922 & 10.560 & 2 & $.005^{* *}$ \\
\hline
\end{tabular}


regardless of physical functional status, depressive symptoms, and self-rated health models, model 2 exhibits significant improvement on model 1, suggesting significant effects on health due to the added control variables.

Model 3 adds SES variables to model 2, and the likelihood ratio test indicates model 3 has significant improvement on model 2. This implies a significant relationship between SES and health after adjusting for control variables. Model 4 incorporates interaction terms between SES and age based on model 3 in order to examine whether significant SES-health relationship differences exist during different age periods. Based on the likelihood ratio test of model 4 against model 3, we found that there are significant differences in effects of SES on physical functional status during different age periods; however, no such significant differences in effects of SES on depressive symptoms and self-rated health during different age periods were found. Based on model 4, model 5 considers variables representing area income level in order to examine whether there are significant effects on health due to the characteristics of the area an individual resides in. The likelihood ratio test shows that model 5 has significant improvement over model 4, suggesting a significant relationship between area income level and individual health. Model 6 is a random intercept model based on model 5 regarding the coefficients of SES variables as random variables in order to test for significant differences of SES regression coefficients in different areas. In terms of physical function and depressive symptoms, model 6 has significant improvement over model 5 , indicating that we cannot treat the associations between SES and these two health indicators as fixed since there are significant areal differences. Nevertheless, model 6 for self-rated health does not demonstrate significant improvement on model 5 , suggesting no random variations on the SES-health relationships among different areas.

To further investigate whether SES regression coefficients are affected by the area income level, model 7 incorporates cross-level interaction terms between SES and area income level on model 6. We find that model 7 exhibits significant improvement on model 6 in terms of depressive symptoms and self-rated health, but model 7 does not show significant improvement on the corresponding model $6(P=0.121)$ with regard to physical functional status, though the significance level is close to 0.1 , which implies some degrees of improvement. To summarize, when comparing model 7 to model 6, we claim the SES-health relationship is affected by the area income level. If the wealth condition of the area where an individual resides is different, the corresponding SES-health association could also be different.

(ii) The age pattern of SES-health relationship Table 2 presents the estimates of model 7 for physical functional status, depressive symptoms, and self-rated health. The models include SES-age interactions and SESarea income-level interactions; the age and area income level variables are centered by their means. ${ }^{9}$ This implies that the regression coefficients of the two dummy variables, namely the highest and medium SES, should be interpreted as the health difference among different SES groups with area income level 4762.47 yuan and aged 60. We observe that the higher the socioeconomic status, the better the individual health, the fewer the depressive symptoms, and the lower the probability of self-rated health as "not good." For instance, the medium-SES group is on average 1.032 lower 
Table 2 Multilevel model estimates of the relationship between socioeconomic status and health

\begin{tabular}{|c|c|c|c|}
\hline & Physical functional status & Depressive symptoms & Self-rated health \\
\hline \multicolumn{4}{|l|}{ Fixed effects } \\
\hline Intercept & $20.306^{* * *}(.200)$ & $15.582^{* * *}(.209)$ & $-1.089^{* * * *}(.069)$ \\
\hline Female (male) & $1.362^{* * *}(.148)$ & $1.933^{* * *}(.138)$ & $.437^{* * *}(.059)$ \\
\hline Separated (married) & $-.292(.257)$ & $.647^{* * *}(.239)$ & $-.039(.102)$ \\
\hline Widowed (married) & $.317^{*}(.188)$ & $1.203^{* * *}(.175)$ & $-.158^{* *}(.072)$ \\
\hline Quit (never smoking) & $1.216^{* * *}(.219)$ & $.524^{* * *}(.203)$ & $.525^{* * *}(.083)$ \\
\hline Always smoking (never smoking) & $-.452^{* * *}(.157)$ & $.069(.146)$ & $-.045(.063)$ \\
\hline Age (centered by 60 ) & $.218^{* * *}(.008)$ & $.044^{* * *}(.007)$ & $.026^{* * *}(.003)$ \\
\hline Medium SES (low SES) & $-1.032^{* * *}(.161)$ & $-1.025^{* * *}(.156)$ & $-.384^{* * *}(.060)$ \\
\hline High SES (low SES) & $-1.512^{* * *}(.254)$ & $-2.469^{* * *}(.248)$ & $-.649^{* * *}(.112)$ \\
\hline County income level (centered by mean) & $-.168^{* * *}(.055)$ & $-.353^{* * *}(.058)$ & $-.095^{* * *}(.017)$ \\
\hline Age $\times$ medium SES & $-.058^{* * *}(.014)$ & $.005(.013)$ & $.009(.006)$ \\
\hline Age $\times$ high SES & $-.075^{* * *}(.018)$ & $-.022(.017)$ & $-.0003(.008)$ \\
\hline County income level $\times$ medium SES & $.084(.057)$ & $.138^{* *}(.056)$ & $.059^{* * *}(.020)$ \\
\hline County income level $\times$ high SES & $.135^{* *}(.066)$ & $.283^{* * *}(.065)$ & $.067^{* * * *}(.025)$ \\
\hline \multicolumn{4}{|l|}{ Random effects } \\
\hline \multicolumn{4}{|l|}{ Level 2 (county level) } \\
\hline Variance (intercept) & 2.192 & 3.112 & .131 \\
\hline Covariance (intercept, medium SES) & -1.288 & -1.248 & -.002 \\
\hline Variance (medium SES) & .757 & .855 & .034 \\
\hline Covariance (intercept, high SES) & -1.552 & -1.913 & -.078 \\
\hline Covariance (medium SES, high SES) & .912 & 1.094 & .045 \\
\hline Variance (high SES) & 1.098 & 1.478 & .103 \\
\hline \multicolumn{4}{|l|}{ Level 1 (individual) } \\
\hline Variance & 34.016 & 29.183 & 1.000 \\
\hline
\end{tabular}

${ }^{*} p<0.1 ;{ }^{* *} p<0.05 ;{ }^{* * *} p<0.01$

in the physical functional score than the lowest-SES group, and the highest-SES group is on average 1.512 lower in the physical functional score than the lowest-SES group. In regard to the depressive symptom score, the medium-SES group and the highest-SES group are 1.025 and 2.469 lower than the lowest SES group. With respect to self-rated health, the medium-SES group is significantly less likely to report as "not good" than the lowest-SES group; the odds of reporting as "not good" from the former group is only around $68 \%$ of the odds from the latter group; the odds of reporting as "not good" from the highest-SES group is only around $52 \%$ of the odds from the lowest-SES group.

Table 2 indicates that the SES-age interaction terms in the model for physical functional status are statistically significant; this reveals the significant age pattern of the relationship between SES and physical function. To further investigate the age pattern of the SES-health relationship, we computed the health scores for different SES groups within different age periods based on model 7 (predicted values). As indicated in Fig. 1, at around age 45, the predicted scores of physical function among different SES groups are quite close. As age increases, especially after 60, the gaps between the lowest-SES 

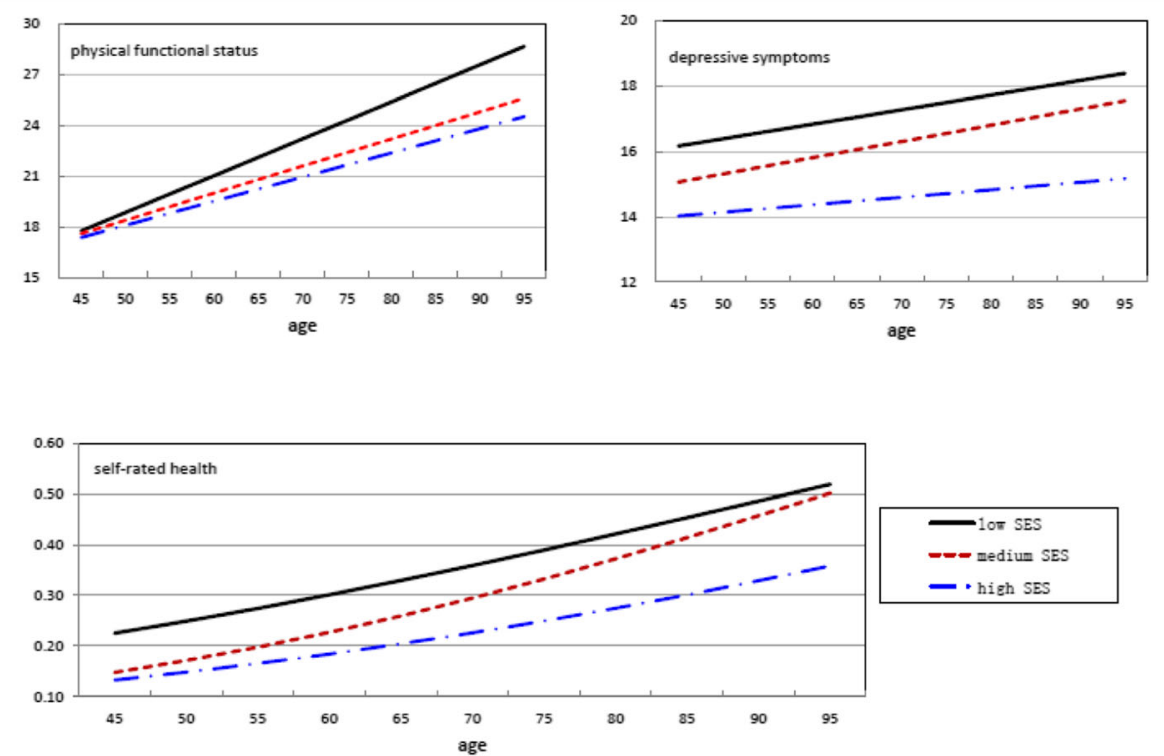

Fig. 1 Health status of different SES groups within different ages (predicted values)

group and the medium- or highest-SES group gradually grow wider, but the differences between the medium- and the highest-SES group fail to suggest significant changes due to age. Meanwhile, as age increases, the rate at which the physical functional score increases among the lowest-SES group (the slope of the line), namely the speed at which the physical functional conditions decreases, is greater than that among the medium- or the highest-SES group. This is the primary reason why the differences of the physical functional score among different SES groups grow larger as age increases.

As indicated in Table 2, the SES-age interaction terms are not significant $(P>0.1)$ in the depressive symptom model. Overall, the differences in depressive symptoms among different SES groups are not affected by age. We see from Fig. 1 that the two lines for the lowest- and the medium-SES groups are almost parallel, suggesting no significant differences of depressive symptoms among the lowest-SES group and among the medium-SES group as age increases. In addition, Fig. 1 shows that the difference between the lowest- and the highest-SES groups and the difference between the medium- and the highest-SES groups tend to expand as age increases; however, this may be a consequence of sampling error.

In the model for self-rated health, the interaction term between the medium SES and age is not statistically significant at the 0.05 level, but it is borderline significant at the 0.1 level $(P=0.11)$. In Fig. 1 , we see that the self-rated health differences between the lowest- and the medium-SES groups gradually decrease with increasing age; their self-rated health reports are fairly close especially during the elderly period. Table 2 also indicates that the interaction between the highest-SES group and age is not statistically significant. Fig. 1 shows there are no significant variations of self-rated health differences between the lowest- and the highest-SES 
groups as age increases. However, Fig. 1 suggests a trend of expanding self-rated health differences between the medium- and the highest-SES groups with increasing age.

In summary, significant differences in the physical functional status exist among different SES groups, and these differences expand with increasing age. With respect to depressive symptoms there are significant differences among different SES groups, but overall these differences do not vary significantly as age increases. However, general conclusions cannot be made on the trend of differences in self-rated health due to increasing age.

(iii) The area pattern of the SES-health relationship

To examine the potential differences between SES-health associations within different areas, we regard the parameters of the SES variables as random coefficients. Compared with the random intercept model, the random coefficient model comes with five new parameters, the SES regression coefficients and their variances or covariance. In the model for physical function, Table 2 indicates that the variance of the medium-SES coefficient is estimated to be 0.757 while the variance of the highest-SES coefficient is 1.098. Based on this, we infer the 95 percent confidence intervals for the medium- and the highest-SES coefficients as [-2.737, 0.673] and [-3.566, 0.542], respectively. Similarly, in the model for depressive symptoms, the regression coefficient of the medium-SES variable lies approximately between -2.838 and 0.788 , whereas the regression coefficient of the highest-SES variable lies approximately between -4.852 and -0.086 . In the model for self-rated health, the regression coefficient of the medium-SES variable lies approximately between -0.745 and -0.023 , whereas the regression coefficient of the highest-SES variable lies approximately between -1.278 and -0.020 . Therefore, the effects of SES on health manifest apparent fluctuations within different areas, that is, the SES-health relationship is likely to be influenced by specific socioeconomic characteristics among different areas.

To further examine how areal socioeconomic characteristics influence the SES-health association, we introduced interaction terms between the SES variables and the area income level. As shown in Table 2, all the interaction terms of the SES variable and area income level are positive, suggesting that the regression coefficients of SES variables are smaller in areas with higher income level, that is, health differences among different SES groups decrease. As implied in Fig. 2, with the improvement of the area income level, individuals' health improves regardless of the SES group they belong to. Nevertheless, the rates at which the health of different SES groups increase with increasing age differ. In general, the area income level has a greater impact on the health status of the lowest-SES group than that of the other higher-SES groups. With the improvement of the area income level, the health gaps among different SES groups gradually grow narrower.

As indicated in Fig. 2, the differences in terms of physical function among different SES groups are greatest in areas with lower income level; these differences gradually shrink with increasing area income level until the physical function scores are fairly close to each other; in areas with higher income level, the differences in physical function among different SES groups almost disappear. Similar patterns are observed for depressive symptoms; in other words, the 

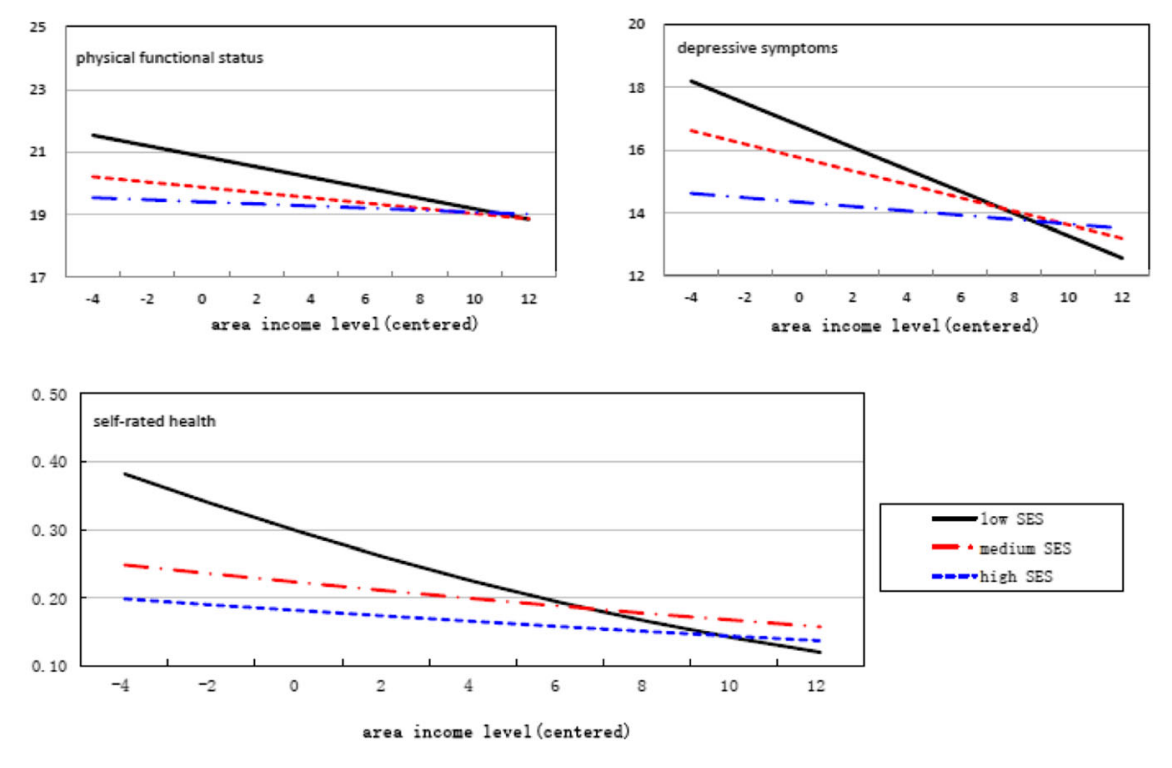

Fig. 2 Health status of different SES groups within different area income levels (predicted values)

differences among different SES groups are greatest in areas with lower income level; these differences gradually shrink with increasing area income level until the depressive symptom scores are fairly close to each other. In the wealthiest areas, the depressive symptom scores of the lowest-SES group fall below the scores of the other two higher-SES groups. The influence of area income level is more significant on depressive symptoms than its influence on physical functional status.

It should be noted that in areas with a lower income level, the probability of self-rated health as "not good" is higher among the lowest-SES group than among the medium-SES group. This probability among the lowest-SES group gradually becomes smaller than that among the medium-SES group when the area income level increases to a certain amount (for example, above 10,000 yuan); similar phenomena are found between the lowest- and the highest-SES groups. Nonetheless, further investigation reveals that the above results may be a consequence of sampling errors and hence not representative of statistical significance. For instance, if we re-estimate model 7 by restricting it to samples with median annual household income per capita above 10,000 yuan within urban areas, we fail to report statistically significant interaction terms of the SES variables and area income level. This further illustrates that no significant differences in self-rated health exist among different SES groups in wealthier areas.

\section{Conclusion and discussion}

Compared to previous research, the samples used in the present study include not only the middle-aged group between 45 to 59 years old but also a fairly number of elderly people over 60 so that we could evaluate the impact of SES on health at different age periods. In addition, the current study makes use of the multilevel data structure, which not only ensures effectiveness in estimating regression coefficients but also allows for the examination of interactions between the micro and macro variables. Specifically, we 
can investigate whether there is an association between the effects of SES variables on health (i.e., regression coefficients) and the area-level characteristics. We selected three health indicators, enabling us to compare the differences between the impacts of SES on different health indicators.

The present study shows that within the Chinese elderly group, the higher-SES group has better physical functional status, fewer depressive symptoms and better self-rated health, which is consistent with previous findings (Lowry and Xie 2009; Rostad et al. 2009; Liu and Zhang 2004; Beydoun and Popkin 2005). This further illustrates that health inequality is a worldwide problem. WTO and their experts have always emphasized that the primary reason for the unequal distribution of health status is social class, instead of viruses or pathogens. Just as with other social inequalities, health inequality will be detrimental to the entire society if it continues to worsen. Thus, it should be an important goal for the structural reform of medical treatment and public health to eliminate such health inequality.

Another important focus of the present research is to investigate the age pattern of the SES-health relationship. Based on our results, we cannot make general statements regarding whether the health differences among different SES groups will expand or shrink as age increases since it depends on the choice of the health indicators. For example, during the underage period, differences in physical function are rather small among different SES groups, but as age increases, these differences become larger, a conclusion supporting the "Cumulative Advantage Hypotheses" (Ross and Wu 1996; Lowry and Xie 2009). Differences in self-rated health between the medium- and the highest-SES groups further expand as age increases, supporting the "Cumulative Advantage Hypotheses" to some extent. Nevertheless, the self-rated health differences between the lowest- and the medium-SES groups shrink with increasing age, which supports the "Convergence Hypotheses" (House et al. 1990, 1994). In terms of depressive symptoms, no significant variations are found for differences among different SES groups as age increases.

The problem among different SES groups of widening gaps in physical function with increasing age could be explained in the following two ways. (1) Objective health indicators such as physical functional status will more likely be greatly affected by SES. Individual health endowment could compensate for weaknesses of SES at a younger age to some extent, but as age increases individual health endowment gradually declines. Individuals' socioeconomic conditions can affect this process of decline to some extent. Due to their advantages in educational attainment, occupation, and income, people with better socioeconomic conditions can acquire more information, knowledge, and material resources than people with worse socioeconomic conditions to prevent their physical function from declining; when physical function declines, the higher-SES group has more advantages in terms of physical function recovery than the lower-SES group. (2) The effects of SES on health are not temporary but sustained and accumulative. The SES within each period in the lifecycle can make an important impact on health in the current or next period. For instance, parents' SES has an important influence on their offspring during childhood while the health of offspring affects their educational attainment and their occupational class during adulthood, and in their adulthood, SES further impacts their health behaviors and psychological orientation. Thus, during the elderly period, the accumulated health risk factors over all life periods 
make a substantial impact on health. After incubating for a long period, some diseases that are dormant at a younger age will be present during the elderly period. Based on this, when developing public health services and health care policies, the elderly group with lower SES should be prioritized, especially elderly people of advanced age, and at the same time the various mechanisms by which the SES of different life periods influences health should be considered.

The present study also reveals that self-rated health differences between the lowestand the medium-SES groups shrink to some extent with increasing age, which is probably related to the measuring properties of self-rated health. Compared to the objective physical functional indicator, self-rated health and depressive symptoms are based on a subjective cognitive process and are characterized by an interactive process between objective health status and the subjective feelings (Jylhä 2009). People with different SES may have different referencing standards when evaluating their own health, and they may differ in their understanding of their own health status. As a result, the same self-rated health may imply different "objective" health status (Dowd and Zajacova 2010). In many cases, respondents may not even know their true health status. For instance, people with a lower educational level or from areas with less-developed public health conditions are likely to report better than actual health status due to lack of knowledge of their physical health risks. Previous studies point out that most elderly people have a positive attitude toward their health. Underlying factors can include the fact that elderly people mainly compare themselves to their peers when rating their health, regard living to an old age as an achievement, or adopt a general definition for life quality and deem themselves competent for the environmental requirements (Hooyman and Kiyak 2011). Thus as age increases, differences in objective health status among different SES groups may expand, though the lowest-SES group holds an optimistic attitude toward their health status, compensating for differences in objective health status to some extent and creating a trend of narrowing self-rated health differences between the lowest- and the medium-SES groups. However, due to the wide gap in health between the lowest- and the highest-SES groups, it is not possible to completely compensate for health differences even though people from the lowest-SES group have an optimistic attitude toward their health differences. The expanding trend of self-rated health differences between the medium- and the highest-SES groups with increasing age is related to not only the Cumulative Advantage Hypothesis mentioned previously but also the features with which the medium-SES group rates their health. Compared to the lowest-SES group, the medium-SES group may have higher health expectations and more channels to acquire health information; therefore, they are less positive about their self-rated health.

Additionally, the current study shows that there are significant differences in the associations between SES and the three selected health indicators within different areas; the county income level has a significant impact on individual health status and significantly adjusts the SES-health association. As the level of wealth in an area improves, the health differences among different SES groups shrink. The reason is that when an area is wealthy, the government increases public spending and is more capable of improving the county's public services such as education and basic medical health care; these improve the health status of the lower-SES group (Feng and Yu 2007). With the improvement of an area's economy, those individuals who get rich earlier will increase 
their demand for their own health, prompting some medical institutions to introduce more-advanced medical technology and thus improving the health of the general public. As a result, the health differences among different SES groups grow close to each other. In contrast, in relatively poorer areas health resources and spending are relatively scarce, making it more difficult for the lower-SES group to access public health resources and services, and further expanding the inequality in access to and availability of public health resources among different SES groups. As a result, future public health service and policy making or reform should consider how to increase investment in public health resources and services in poorer areas so that people from areas with different income levels could share the same public health services, hence narrowing the health inequality gap in different areas.

Our study has the following three limitations. (1) Since our study uses cross-sectional data, it is not possible to distinguish between age effects and cohort effects. The fact that we attribute the age pattern of the SES-health relationship to effects due to the increase of age but not effects due to cohort membership is based on the following assumption: the age pattern of the SES-health relationship does not significantly change among different cohorts. Nonetheless, there are studies that point out increasingly stronger age patterns in the SES-health relationship (Lynch 2003); in young cohorts education has a stronger effect on health (Lauderdale 2001). Hence, the age pattern of the SES-health relationship presented in the current study includes not only effects due to age but also cohort effects. (2) The present study suffers from an innate issue, namely that we are not able to distinguish the causal directions between the SES and health status. In other words, health status may affect SES, and people with better health status could gain opportunities to migrate to higher-SES groups; in contrast, people with worse health status may move downward in social class (Dahl 1996; West 1991), therefore widening the health gap among different SES groups. (3) The current study may suffer from selection bias in regard to physical function. Given that the probability of exclusion from the study of the severely paralyzed is higher than that for people with better physical functional status, namely the sampled respondents all maintain a certain level of physical function, it is likely that we overestimated the physical functional status for the current sample while underestimating differences in physical functional status among different SES groups.

In conclusion, this study further confirms the evidence of social inequality among different SES groups and the variations of this inequality during different age periods and over different areas. Considering the SES-health relationship is affected by the socialpolitical-economic background, with the rapid expansion of the economy and the profound change in China's social structure, we should continue investigating and analyzing Chinese health inequality and its trend of development under the new social context.

\section{Endnotes}

${ }^{1}$ More details on the data set can be found at the official Web site: http:// charls.ccer.edu.cn/zh-CN- CN.

${ }^{2}$ There are 20 measurement items in total for physical function in CHARLS; three items with lower reliability were deleted in this study after a reliability test was performed (Cronbach's $\alpha$ ). 
${ }^{3}$ One item was deleted based on the reliability test (Cronbach's $\alpha$ ).

${ }^{4} \mathrm{~A}$ basic principle in combining categories is to combine similar categories and ensure differences among combined categories. In regard to self-rated health, physical health and depressive symptoms are significantly associated; we computed the scores for different items of self-rated health in terms of physical function and depressive symptoms and found the difference between self-rated "ordinary" and self-rated "good" was smaller than that between self-rated "ordinary" and self-rated "not good." We therefore combined self-rated "ordinary" and self-rated "good."

${ }^{5}$ Including not graduating from elementary school and similarly for the following categories.

${ }^{6}$ Many previous research works also divided occupations into two or three categories (e.g., Liu and Zhang 2004).

${ }^{7}$ We had two considerations for the classification: one is that we used the quartiles of annual family income per capita to determine the approximate boundaries for each group; the other is we chose 2300 yuan as the upper limit for the first group given, that the Chinese poverty line is 2300 yuan.

${ }^{8}$ Details can be found at http://cran.r-project.org/web/packages/lme4/.

${ }^{9}$ Centering was performed with respect to the mean of each variables; i.e., the mean was subtracted in the original variable.

Competing interests

The authors declare that they have no competing interests.

\section{Acknowledgements}

This study is the progressive result from of the National Social Science Funding Project "A study on the problem and the mechanism of health inequality among the elderly group" (Project Approval Number: 14BRK012). We thank the anonymous referees for comments and suggestions.

Received: 25 November 2015 Accepted: 6 April 2016

Published online: 21 April 2016

References

Bassuk, Shari S, Lisa F. Berkman, and Benjamin C. Amick. 2002. Socioeconomic status and mortality among the elderly: Findings from four US communities. American Journal of Epidemiology 155(6): 520-533.

Black, Douglas. 1981. Inequality in health. British Medical Journal (Clinical Research Edition) 282(6274): 1468.

Beckett, Megan. 2000. Converging health inequalities in later life-an artifact of mortality selection? Journal of Health and Social Behavior: 106-19.

Beydoun M A, Popkin B M. 2005. The impact of socio-economic factors on functional status decline among community-dwelling older adults in China [J]. Social science \& medicine 60(9): 2045-2057.

Dachs, J, W. Norberto, Marcela Ferrer, Carmen Elisa Florez, Aluisio J.D. Barros, Rory Narváez, and Martín Valdivia. 2002. Inequalities in health in Latin America and the Caribbean: Descriptive and exploratory results for self-reported health problems and health care in twelve countries. Revista Panamericana de Salud Pública 11(5): 335-355.

Dahl, Espen. 1996. Social mobility and health: Cause or effect?". British Medical Journal 313(7055): 435-436.

Dowd, Jennifer Beam, and Anna Zajacova. 2010. Does self-rated health mean the same thing across socioeconomic groups? Evidence from biomarker data. Annals of Epidemiology 20(10): 743-749.

Dupre, Matthew E. 2008. Educational differences in health risks and illness over the life course: A test of cumulative disadvantage theory. Social Science Research 37(4): 1253-1266.

Ellaway, Anne, Michaela Benzeval, Michael Green, Alastair Leyland, and Sally Macintyre. 2012. Getting sicker quicker: Does living in a more deprived neighbourhood mean your health deteriorates faster?". Health \& Place 18(2): 132-137.

Evans, Gary W., and Elyse Kantrowitz. 2002. Socioeconomic status and health: The potential role of environmental risk exposure. Annual Review of Public Health 23(1): 303-331.

Feinstein, Jonathan S. 1993. The relationship between socioeconomic status and health: A review of the literature. The Milbank Quarterly 71(2): 279-322.

Feng, J, and Y. Yu. 2007. The income gap and health in China rural areas. Economic Research Journal 1: 79-88.

Glied, Sherry, and Adriana Lleras-Muney. 2008. Technological innovation and inequality in health. Demography 45(3): 741-761.

Guo, Y, and Z. Xie. 2009. Bridging the gap within a generation-the theory of the determinant for healthy societies and relevant international experience. Journal of Peking University (Health Sciences) 41(2): 125-128

Hooyman, NR, and H. Asuman Kiyak. 2011. Social gerontology: A multidisciplinary perspective. Boston: Allyn \& Bacon. 
House, James S, Ronald C. Kessler, and A. Regula Herzog. 1990. Age, socioeconomic status, and health. The Milbank Quarterly 68(3): 383-411.

House, James S, James M. Lepkowski, Ann M. Kinney, Richard P. Mero, Ronald C. Kessler, and A. Regula Herzog. 1994. The social stratification of aging and health. Journal of Health and Social Behavior 35(3): 213-234.

Huurre, Taina, Ossi Rahkonen, Erkki Komulainen, and Hillevi Aro. 2005. Socioeconomic status as a cause and consequence of psychosomatic symptoms from adolescence to adulthood. Social Psychiatry and Psychiatric Epidemiology 40(7): 580-587.

Idler, Ellen L, and Yael Benyamini. 1997. Self-rated health and mortality: A review of twenty-seven community studies. Journal of Health and Social Behavior 38(1): 21-37.

Jylhä, Marja. 2009. What is self-rated health and why does it predict mortality? Towards a unified conceptual model. Social Science \& Medicine 69(3): 307-316.

Lantz, Paula M, James S. House, James M. Lepkowski, David R. Williams, Richard P. Mero, and Jieming Chen. 1998. Socioeconomic factors, health behaviors, and mortality: Results from a nationally representative prospective study of US adults. The Journal of the American Medical Association 279(21): 1703-1708.

Lauderdale, Diane S. 2001. Education and survival: Birth cohort, period, and age effects. Demography 38(4): 551-561.

Liu, L, and J. Tang. 2004. The effects of socioeconomic status on residents' health equality. Chinese Health Economics 23(6): 40-42.

Liu, Guiping, and Zhen Zhang. 2004. Sociodemographic differentials of the self-rated health of the oldest-old Chinese. Population Research and Policy Review 23(2): 117-133.

Lowry, Deborah, and Yu Xie. 2009. Socioeconomic status and health differentials in China: Convergence or divergence at older ages? Population Studies Center, University of Michigan.

Lynch, Scott M. 2003. Cohort and life-course patterns in the relationship between education and health: A hierarchical approach. Demography 40(2): 309-331.

Mackenbach, Johan P, Anton E. Kunst, Adrienne E.J.M. Cavelaars, Feikje Groenhof, and Jose J.M. Geurts. 1997. Socioeconomic inequalities in morbidity and mortality in Western Europe. The EU Working Group on Socioeconomic Inequalities in Health. The Lancet 349(9066): 1655-1659.

Meng, Q. 2007. The Chinese public health and medical care reformation and health equality. Chinese Health Economics 26(1): 9-14.

Mirowsky, John, and Catherine E Ross. 2005. Education, cumulative advantage, and health. Ageing International 30(1): 27-62.

Mirowsky, John, and Catherine E. Ross. 2008. Education and self-rated health cumulative advantage and its rising importance. Research on Aging 30(1): 93-122.

Phelan, Jo C, Bruce G. Link, Ana Diez-Roux, Ichiro Kawachi, and Bruce Levin. 2004. Fundamental causes of social inequalities in mortality: A test of the theory. Journal of Health and Social Behavior 45(3): 265-285.

Pickett, Kate E, and Michelle Pearl. 2001. Multilevel analyses of neighbourhood socioeconomic context and health outcomes: A critical review. Journal of Epidemiology and Community Health 55(2): 111-122.

Robert, Stephanie A. 1998. Community-level socioeconomic status effects on adult health. Journal of Health and Social Behavior 39(1): 18-37.

Ross, Catherine E, and Chia-Ling Wu. 1996. Education, age, and the cumulative advantage in health. Journal of Health and Social Behavior 37(1): 104-120.

Rostad, Berit, Dorly J.H. Deeg, and Berit Schei. 2009. Socioeconomic inequalities in health in older women. European Journal of Ageing 6(1): 39-47.

Sun, Q, K. Rao, and Y. Guo. 2003. The impact of different health indicators on health equality index. Chinese Health Statistics 20(4): 197-200

Victora, Cesar G, J. Patrick Vaughan, Fernando C. Barros, Anamaria C. Silva, and Elaine Tomasi. 2000. Explaining trends in inequities: Evidence from Brazilian child health studies. The Lancet 356(9235): 1093-1098.

Wang, F. 2011. Will social mobility help reduce the health inequality?". Sociology Research 2: 78-101.

Wang, F. 2012. Socioeconomic status, lifestyle and health inequality. Society 32(2): 125-143.

West, Patrick. 1991. Rethinking the health selection explanation for health inequalities. Social Science \& Medicine 32(4): 373-384.

Wilkinson, Richard G. 1997. Socioeconomic determinants of health: Health inequalities: Relative or absolute material standards? British Medical Journal 314(7080): 591.

Wu, Xiaogang, and Donald J. Treiman. 2004. The household registration system and social stratification in China: 1955-1996. Demography 41(2): 363-384.

Xie, E. 2009. A study on the income-related health and the inequality of accessing medical care services. Economic Research Journal 2: 92-105.

Yen, Irene H, and S. Leonard Syme. 1999. The social environment and health: A discussion of the epidemiologic literature. Annual Review of Public Health 20(1): 287-308.

Zhu, Haiyan, and Yu Xie. 2007. Socioeconomic differentials in mortality among the oldest old in China. Research on Aging 29(2): 125-143.

Zimmer, Zachary, Napaporn Chayovan, Hui-Sheng Lin, and Josefina Natividad. 2004. How indicators of socioeconomic status relate to physical functioning of older adults in three Asian societies. Research on Aging 26(2): 224-258. 